\title{
Sexual orientation biases attentional control: a possible gaydar mechanism
}

\author{
Lorenza S. Colzato ${ }^{*}$, Linda van Hooidonk ${ }^{1}$, Wery P. M. van den Wildenberg ${ }^{2}$, Fieke Harinck ${ }^{3}$ and \\ Bernhard Hommel ${ }^{1}$ \\ Cognitive Psychology Unit, Leiden Institute for Brain and Cognition, Leiden University, Leiden, Netherlands \\ 2 Amsterdam Center for the Study of Adaptive Control in Brain and Behaviour (Acacia), Psychology Department Amsterdam, University of Amsterdam, Netherlands \\ ${ }^{3}$ Social Psychology Unit, Leiden University, Leiden, Netherlands
}

\section{Edited by:}

Anna M. Borghi, University of Bologna, Italy

\section{Reviewed by:}

Costantini Marcello, University of

Chieti, Italy

Antonino Raffone, Sapienza University

of Rome, Italy

*Correspondence:

Lorenza S. Colzato, Cognitive Psychology Unit, Department of

Psychology, Leiden University, Postbus 9555, 2300 RB Leiden, Netherlands.

e-mail: colzato@fsw.leidenuniv.nl
Homosexuals are believed to have a "sixth sense" for recognizing each other, an ability referred to as gaydar. We considered that being a homosexual might rely on systematic practice of processing relatively specific, local perceptual features, which might lead to a corresponding chronic bias of attentional control. This was tested by comparing male and female homosexuals and heterosexuals - brought up in the same country and culture and matched in terms of race, intelligence, sex, mood, age, personality, religious background, educational style, and socioeconomic situation - in their efficiency to process global and local features of hierarchicallyconstructed visual stimuli. Both homosexuals and heterosexuals showed better performance on global features - the standard global precedence effect. However, this effect was significantly reduced in homosexuals, suggesting a relative preference for detail. Findings are taken to demonstrate chronic, generalized biases in attentional control parameters that reflect the selective reward provided by the respective sexual orientation.

Keywords: sexual orientation, attention, global precedence

\section{INTRODUCTION}

Individuals with a homosexual orientation are often believed to have a "telepathic sixth sense" (Reuter, 2002) for recognizing each other, an ability that is often referred to as gaydar (Shelp, 2002) - a portmanteau of gay and radar. Even though perceivable differences between homosexuals and heterosexuals may not be salient to everyone, some studies revealed subtle but distinctive features that homosexuals tend to share, such as coiffure (Rule et al., 2008), body-movement and gesturing style (Ambady et al., 1999), speech patterns (Linville, 1998), and penile size (Bogaert and Hershberger, 1999). Hence, there is a rich perceptual basis for people to develop a reliable gaydar, and homosexuals are apparently better trained in making use of it.

In the present study, we were not so much interested in the particular perceptual cues that underlie gaydar but we asked whether being a homosexual is associated with systematic changes in attentional control. Most perceptual cues that gaydar needs to consider are relatively specific and local and, thus, require focused attention to reliably pick them up. There is increasing evidence that practicing particular attentional sets can induce chronic attentional biases that generalize to other, practice-unrelated situations.

For instance, individuals growing up in an Asian culture tend to attend to visual scenes and displays more holistically than Westerners do (Nisbett and Masuda, 2003; Nisbett and Miyamoto, 2005). Among other things, this is suggested by their performance on hierarchical stimuli, such as larger letters that are made of smaller letters. People are commonly faster in responding to the global than to the local letter or symbol, an observation that has been coined the global precedence effect (Navon, 1977). This is true for members of Asian and Western cultures alike, but the global precedence effect is more pronounced in Asians than in North Americans - suggesting a relative preference for detail in the latter. This finding has been attributed to cultural learning: while Westerners are raised to develop a relatively independent view about themselves, Easterners have stronger emphasis on social interdependence (Markus and Kitayama, 1991). Acquiring a more independent or more interdependent self-concept might require or suggest attentional sets that are emphasizing more local or more global symbols features, respectively, and thus establish a chronic bias of attentional control processes (Hommel and Colzato, in press).

Recent observations on the impact of religious practice on visual attention provide convergent evidence for this possibility. Colzato et al. (2008) showed that Dutch Calvinists and Dutch atheists, matched for culture, race, sex, age, and intelligence, process global and local visual features differently. A major characteristic of neoCalvinism is the sphere sovereignty principle which, in a nutshell, implies that believers should not judge other groups in society but "mind their own business". This would lead one to expect that Calvinists show a stronger preference for detail and, indeed, the global precedence effect was less pronounced in Calvinists than it was in atheists. A recent study revealed that the global precedence effect is more pronounced in Italian Roman Catholics than in Italian seculars, and in Israeli Orthodox Jews than in Israeli nonbelievers (Colzato et al., 2010 under revision), which fits with the strong emphasis on social solidarity and interdependence in both Catholicism and Orthodox Judaism.

Taken together, these findings imply that the way social/cultural groups shape the behavior of their members, presumably by providing selective reward for norm-fitting, appropriate behavior, poses 
specific demands on attentional control processes (Colzato et al., 2010, under revision; Hommel and Colzato, in press). These specific demands favor some attentional sets over others and thereby induce apparently chronic biases. This does not necessarily imply that individuals would lose their ability to implement other sets, but doing so requires more cognitive effort and, thus, impairs performance. If so, the more pronounced practice in homosexuals to attend to local visual features may lead to a generalized and chronic attentional bias towards local as compared to global features. If this would be the case, homosexuals should show a less pronounced global precedence effect than heterosexuals in the global-local task (Navon, 1977). We tested this hypothesis in male and female individuals with homo- and heterosexual orientation.

\section{MATERIALS AND METHODS PARTICIPANTS}

The sample included 42 healthy subjects ( 25 male and 17 female) between 18 and 30 years of age. Half of the participants reported having a heterosexual orientation and the other half having a homosexual orientation. Members of the two orientation groups were matched for race (100\% Caucasian), culture (100\% Dutch), age, sex, and IQ (measured by Raven's Standard Progressive Matrices), religious background, educational style (Dutch VWO) and socioeconomic situation (see Tables $\mathbf{1}$ and 2). Participants were recruited by advertisements and snowball technique. Written informed consent was obtained from all participants after the nature of the study was explained to them; the protocol and the remuneration arrangements of 8 Euro was approved by the institutional review board (Leiden University, Institute for Psychological Research).

\section{APPARATUS AND STIMULI}

Responses were made by pressing the "Z" or "?" of the QWERTY computer keyboard with the left and right index finger, respectively. The target stimuli were adopted from Huizinga et al. (2006), and consisted of geometric figures. Larger (global) rectangles or squares consisted of smaller (local) rectangles or squares. Global stimuli (i.e., squares or rectangles; $93 \times 93$ pixels or $93 \times 189$ pixels respectively) were composed of many smaller "local" stimuli (i.e., squares or rectangles; $21 \times 21$ pixels or $8 \times 46$ pixels respectively). The space

Table 1 | Demographic characteristics of participants.

\begin{tabular}{lcc}
\hline Sample & Homosexuals & Heterosexuals \\
\hline Sex, $N(\mathrm{M}: F)^{\mathrm{ns}}$ & $21(13: 8)$ & $21(12: 9)$ \\
Age $\left(\right.$ years) $^{\mathrm{ns}}$ & $22.0(2.12)$ & $20.9(2.68)$ \\
Raven IQ $^{\mathrm{ns}}$ & $124.5(3.10)$ & $125.0(4.27)$ \\
AFFECT & & \\
Arousal $^{\mathrm{ns}}$ & $5.43(1.60)$ & $5.48(1.36)$ \\
Mood $^{\mathrm{ns}}$ & $6.29(1.15)$ & $6.62(1.16)$ \\
PERSONALITY & $10.43(1.94)$ & $8.95(3.23)$ \\
Extraversion $^{\mathrm{ns}}$ & $3.53(1.89)$ & $3.10(1.64)$ \\
Psychoticism $^{\mathrm{ns}}$ & $4.10(2.76)$ & $4.52(2.68)$ \\
Neuroticism $^{\mathrm{ns}}$ & & \\
\hline
\end{tabular}

Standard deviations in parentheses.

ns - Non-significant difference. between the local elements of a stimulus was 3 pixels. A global square consisted of 16 small squares or 8 small rectangles; a global rectangle consisted of 32 small squares or 16 small rectangles.

\section{PROCEDURE AND DESIGN}

All participants were tested individually and completed the intelligence test, the affect grid, the Eysenck's personality questionnaire (EPQ-RSS), the sexual orientation grid, and the global-local task.

Individual IQ was determined by means of a 30-min reasoningbased intelligence test (Raven's Standard Progressive Matrices: SPM, Raven et al., 1988). The SPM assesses the individual's ability to create perceptual relations and to reason by analogy independent of language and formal schooling; it is a standard, widelyused test to measure Spearman's $g$ factor and of fluid intelligence in particular.

The affect grid is a single-items scale that measures affect along the dimensions of unpleasant/pleasant feelings and arousal/ sleepiness (Russell et al., 1989). We used the affect grid in order to control for mood given it has been shown that mood influences

Table 2 | Mean scores of sexual orientation grid on 7-point scales.

\begin{tabular}{|c|c|c|}
\hline Scale & Homosexuals & Heterosexuals \\
\hline \multicolumn{3}{|c|}{ SEXUAL ATTRACTION } \\
\hline$|d e a|^{* *}$ & $6.48(0.81)$ & $1.10(0.31)$ \\
\hline Present** & $6.38(0.59)$ & $1.15(0.37)$ \\
\hline Past ${ }^{* *}$ & $4.71(1.35)$ & $1.10(0.31)$ \\
\hline \multicolumn{3}{|c|}{ SEXUAL BEHAVIOR } \\
\hline Ideal $^{* *}$ & $6.48(0.68)$ & $1.05(0.22)$ \\
\hline Present** & $6.52(0.81)$ & $1.00(0.00)$ \\
\hline Past** & $4.95(2.25)$ & $1.05(0.22)$ \\
\hline \multicolumn{3}{|c|}{ SEXUAL FANTASIES } \\
\hline$\left.\right|_{\text {deal }} ^{* *}$ & $6.29(0.90)$ & $1.25(0.64)$ \\
\hline Present** & $6.33(0.91)$ & $1.25(0.64)$ \\
\hline Past** & $5.57(1.63)$ & $1.15(0.49)$ \\
\hline \multicolumn{3}{|c|}{ EMOTIONAL PREFERENCE } \\
\hline$\left.\right|_{\text {deal }} ^{* *}$ & $6.05(1.16)$ & $1.15(0.37)$ \\
\hline Present** & $5.71(1.15)$ & $1.15(0.37)$ \\
\hline Past** & $4.43(1.57)$ & $1.15(0.37)$ \\
\hline \multicolumn{3}{|c|}{ LIFESTYLE } \\
\hline$\left.\right|_{\text {deal }} ^{* *}$ & $4.00(0.84)$ & $2.45(1.00)$ \\
\hline Present** & $3.81(1.60)$ & $1.95(0.76)$ \\
\hline Past** & $2.52(1.60)$ & $1.70(0.86)$ \\
\hline \multicolumn{3}{|c|}{ SELF-IDENTIFICATION } \\
\hline Ideal** & $6.51(0.75)$ & $1.15(0.37)$ \\
\hline Present** & $6.29(0.64)$ & $1.15(0.37)$ \\
\hline Past** & $4.14(1.80)$ & $1.10(0.31)$ \\
\hline \multicolumn{3}{|c|}{ SOCIAL PREFERENCE } \\
\hline$|d e a|^{\text {ns }}$ & $4.48(0.75)$ & $4.00(1.12)$ \\
\hline Present $^{\text {ns }}$ & $4.51(1.24)$ & $4.25(1.16)$ \\
\hline Past ${ }^{\text {ns }}$ & $4.48(1.33)$ & $4.10(1.89)$ \\
\hline
\end{tabular}

Standard errors are presented within parentheses.

ns - Non-significant difference.

Significant group difference ${ }^{* *} p<0.01$. 
the attentional scope: more positive mood leads to a more global attention scope (Rowe et al., 2007). Participants were asked to put a mark in the grid to indicate their feelings at the present moment. The vertical dimension represents the degree of arousal vs. sleepiness while the horizontal dimension represents unpleasant vs. pleasant feelings.

The EPQ-RSS questionnaire consists of 48 yes/no questions that measure extraversion, neuroticism and psychoticism traits of personality (Eysenck et al., 1985). We controlled for personality traits because it has been shown that more extravert individuals tend to show an enhanced global scope compared to neurotic individuals (Kossowska and Necka, 1994).

The Dutch translation of the sexual orientation grid (Klein et al., 1985) was used in order to quantify sexual orientation. This scale consists of seven component variables that indicate a person's sexual orientation at three different points: their past, their present and their ideal. Subjects have to score each of the 21 resulting combinations on a 7 -point scale ranging from $1=$ other sex only to $7=$ same sex only for the variables sexual attraction, sexual behavior, sexual fantasies, emotional preference and social preference. For the variables lifestyle and self-identification the scale is ranging from $1=$ heterosexual only to $7=$ gay/lesbian only.

In the global-local task (cf., Huizinga et al., 2006), participants responded to randomly presented rectangles or squares by pressing a left or right response button, respectively. A cue indicated to which dimension (global or local) the participants should respond. Cues that signaled the global (local) dimension consisted of a large (small) square, presented at one side of the target stimulus, and a large (small) rectangle, presented at the other side of the target stimulus. The color of cues and target was red. They remained on the screen until a response was given or $3500 \mathrm{~ms}$ had passed. The time interval between presentation of the cue and of the target stimulus was $500 \mathrm{~ms}$. The interval between the response and the presentation of the cue was fixed at $1000 \mathrm{~ms}$. Participants responded to the global shape in one practice block and to the local shape in another practice block; block order was randomized and each block comprised of 50 experimental trials. In the third experimental block, consisting of 150 trials, participants alternated between predictable sequences of four "local" and four "global" trials.

\section{STATISTICAL ANALYSIS}

Independent samples $t$-tests were performed to test age, mood, personality traits and IQ differences between the groups. Following Huizinga et al. (2006) and Colzato et al. (2008), median reaction times (RTs) and square root error percentages were analyzed by means of ANOVAs using Target Level (global vs. local) as within- and Group (Homosexuals vs. Heterosexuals) as betweenparticipants factor. A significance level of $p<0.05$ was adopted for all tests.

\section{RESULTS}

\section{SEXUAL ORIENTATION GRID}

Analysis of the responses to the sexual orientation grid (Klein et al., 1985) revealed significant differences between homosexual and heterosexual participants on the variables Sexual Attraction, Sexual Behavior, Sexual Fantasies, Emotional Preference, Lifestyle and SelfIdentification. All variables measured the past (more than 1.5 years ago), present and personal ideal situation. No significant differences were found on Social Preference for their past $t(40)=0.92, p=0.36$, present, $t(40)=0.73, p=0.47$, and ideal situation, $t(40)=1.60$, $p=0.12$. The Social Preference item regards the sex of the people with whom the participants socialize. It is therefore not surprising that the Social Preference variable revealed no significant difference between homosexuals and heterosexuals (see Table 2 for an overview).

\section{GLOBAL-LOCAL TASK}

Heterosexuals tended to be faster but less accurate than homosexuals, but the main effect of Group did not reach significance in RTs or error rates (ERRs), $p$-values $>0.25$. The RT analysis yielded a main effect for Target Level, $F(1,40)=89.45, p<0.0001$, MSE $=674.69$, $\eta^{2} p=0.69-$ the global precedence effect (Navon, 1977): global targets were responded to faster than local targets. However, as expected, the size of this effect varied with Group, $F(1,40)=6.17$, $p<0.05, \mathrm{MSE}=674.69, \eta^{2} p=0.13$, (see Figure 1). Homosexuals showed a less pronounced, but still significant, $F(1,20)=21.14$, $p<0.001, \mathrm{MSE}=775.68, \eta^{2} p=0.51$, global precedence effect than heterosexuals, $F(1,20)=83.9, p<0.0001, \mathrm{MSE}=573.41, \eta^{2} p=0.81$, (see Table 3). Analysis of the ERRs also revealed a main effect for Target Level, $F(1,40)=12.93, p<0.01, \mathrm{MSE}=30.23, \eta^{2} p=0.23$, due to more errors being made on local targets, but this effect was not reliably modified by Group.

\section{DISCUSSION}

The aim of this study was to investigate whether people's sexual orientation was associated with a generalized attentional bias toward a local level of perceptual analysis. Overall, participants responded faster to global than to the local features of visual stimuli, replicating the well-known global precedence effect (Navon, 1977). As predicted, however, this effect was modified by sexual orientation: Homosexuals showed a significantly less pronounced global precedence effect than heterosexuals, suggesting that homosexuals indeed adopt a more analytic (local) perceptual style.

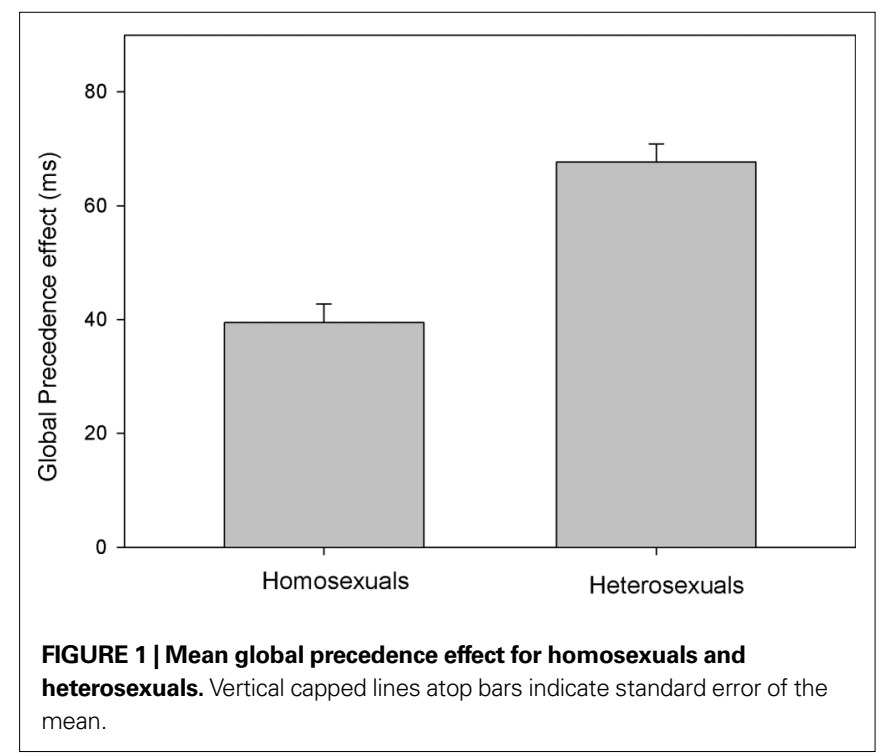


Table 3 | Mean reaction times (RTs, in $\mathrm{ms}$ ) and error rates (ERRs, in \%) for responses to globally and locally defined targets and the global precedence effect (local-global).

\begin{tabular}{lllll}
\hline & \multicolumn{2}{c}{ Homosexuals } & \multicolumn{2}{c}{ Heterosexuals } \\
\hline & RT & ERR & RT & ERR \\
\hline Global targets & $414(65)$ & $5.2(5.8)$ & $350(44)$ & $5.1(3.8)$ \\
Local targets & $454(64)$ & $7.8(6.8)$ & $418(60)$ & $11.2(7.3)$ \\
Global precedence & $40^{* *}$ & 2.5 & $68^{* *}$ & 6.1 \\
\hline
\end{tabular}

Standard errors in parentheses.

Significant group difference ${ }^{*} p<0.01$.

Adopting such a perceptual style presumably increases the likelihood to detect perceptual cues indicative of homosexual orientation, which again facilitates finding like-minded, social peers, and potential friends and sex mates. Accordingly, adopting a local attentional set is likely to be socially rewarded, which seems to imprint this set to a degree that it generalizes to non-social situations, such as responses to geometric shapes. Consistent with our hypothesis, Ambady et al. (1999) showed that homosexuals are more accurate in detecting the sexual orientation of other people shown in pictures and brief video clips than heterosexual participants. Given that homosexuals no longer outperformed heterosexuals when longer video clips were shown, they are unlikely to have privileged knowledge about perceptual cues correlated with sexual orientation. Rather, they seem to be better (i.e., faster and/ or more efficient) in extracting these cues from the visual environment. Our findings suggest that this is because their attentional set might be better tuned to pick up these commonly locally defined cues. However, it remains to be seen whether this attentional bias is sufficient to establish a reliable gaydar.

We speculate that homosexuals are more likely to adopt chronic local attentional sets because these sets facilitate the processing of behaviorally relevant cues and are, thus, rewarded. An alternative interpretation might be based on the social situation homosexuals are finding themselves in (Derryberry and Reed, 1998). Given that

\section{REFERENCES}

Ambady, N., Hallahan, M., and Conner, B. (1999). Accuracy of judgments of sexual orientation from thin slices of behavior. J. Pers. Soc. Psychol. 77, 538-547.

Boduroglu, A., Shah, P., and Nisbett, R. E. (2009). Cultural differences in allocation of attention in visual information processing. J. Cross Cult. Psychol. 40, 349-360.

Bogaert, A. F., and Hershberger, S. (1999). The relation between sexual orientation and penile size. Arch. Sex. Behav. 28, 213-221.

Colzato, L. S., van den Wildenberg, W. P. M., and Hommel, B. (2008). Losing the big picture: how religion may control visual attention. PLoS ONE 3, e3679. doi:10.1371/journal.pone.0003679.

Derryberry, D., and Reed, M. A. (1998). Anxiety and attentional focusing: Trait, state and hemispheric influences. Pers. Individ. Dif. 25, 745-761.

Eysenck, S. B. G., Eysenck, H. J., and Barrett, P. (1985). A revised version of the psychoticism scale. Pers. Individ. Dif. 6, 21-29.

Hommel, B., and Colzato, L. S. (in press). Religion as a control guide: on the impact of religion on cognition. Zygon J. Relig. Sci.

Huizinga, M., Dolan, C. V., and van der Molen, M. W. (2006). Age-related change in executive function: developmental trends and a latent

homosexuals are a minority in our society, being a homosexual might be associated with enhanced anxiety - an affective state that has been reported to induce a narrower attentional scope (Rowe et al., 2007). In fact, homosexuals are more often confronted with lifetime and day-to-day discrimination than heterosexuals (Mays and Cochran, 2001) and it seems plausible that this causes increased levels of psychological distress in homosexuals (Meyer, 1995). Indeed, there is evidence that homosexual men experience more social-interaction anxiety than heterosexual men, which might be due to the experience of stigmatization and discrimination (Pachankis and Goldfried, 2006). However, we were unable to find any indication of greater anxiety or arousal in our homosexual participants: the two groups did not differ with regard to a single scale in either affective state or personality and even the numerical differences indicate lower, rather than higher neuroticism and arousal scores in homosexuals. Even though these observations do not contradict the finding of more social anxiety in homosexual individuals, they rule out a stress-related account of the local attentional bias.

From a more general perspective, our findings add to previous observations that being a member of a particular social group seems to shape cognitive-control operations in specific ways - whether this group is defined by shared culture (Nisbett and Masuda, 2003; Nisbett and Miyamoto, 2005; Boduroglu et al., 2009), religious practice (Colzato et al., 2008; Colzato et al., 2010 under revision) or, as the present study suggests, shared sexual orientation. Even though more research is necessary on the establishing conditions and processes, it makes sense to assume that social groups provide selective reward for particular types of behavior, which again imply, suggest, or require the adoption of particular control strategies or cognitive sets (Colzato et al., 2010 under revision; Hommel and Colzato, in press). Once a strategy or set is sufficiently strengthened, it may generalize to other, actually unrelated situations and bias information processing accordingly. There is no reason to assume that this scenario is restricted to culture, religion, and sexual orientation, suggesting that our observations reflect a very general mechanism. As implied by Nisbett and Miyamoto (2005), any kind of socially guided practice may have the same potential of inducing systematic cognitive biases.

variables analysis. Neuropsychologia 44, 2017-2036.

Klein, F., Sepekoff, B., and Wolf, T. J. (1985). Sexual orientation: a multivariable dynamic process. J. Homosex. $11,35-49$.

Kossowska, M., and Necka, E. (1994). Do it your own way: cognitive strategies, intelligence, and personality. Pers. Individ. Dif. 16, 33-46.

Linville, S. (1998). Acoustic correlates of perceived versus actual sexual orientation in men's speech. Folia Phoniatr. Logop. 50, 35-48.

Markus, H. R., and Kitayama, S. (1991). Culture and the self: implications for cognition, emotion, and motivation. Psychol. Rev. 98, 224-253.
Mays, V. M., and Cochran, S. D. (2001). Mental health correlates of perceived discrimination among lesbian, gay, and bisexual adults in the United States. Am. J. Public Health 91, 1869-1876.

Meyer, I. H. (1995). Minority stress and mental health in gay men. J. Health Soc. Behav. 36, 38-56.

Navon, D. (1977). Forest before trees: the precedence of global features in visual perception. Cogn. Psychol. 9, 353-383.

Nisbett, R. E., and Masuda, T. (2003). Culture and point of view. Proc. Natl. Acad. Sci. U.S.A. 100, 11163-11170.

Nisbett, R. E., and Miyamoto, Y. (2005). The influence of culture: holistic versus analytic perception. Trends Cogn. Sci. 9, 467-473. 
Pachankis, J. E., and Goldfried, M. R. (2006). Social anxiety in young gay men. J. Anxiety Disord. 20, 996-1015.

Raven, J. C., Court, J. H., and Raven, J. (1988). Manual for Raven's Progressive Matrices and Vocabulary Scales. London: Lewis.

Reuter, D. F. (2002). Gaydar: The Ultimate Insider Guide to the Gay Sixth Sense. New York: Crown.

Rowe, G., Hirsh, J. B., and Anderson, A. K. (2007). Positive affect increases the breath of attentional selection. Proc. Natl. Acad. Sci. U.S.A. 104, 383-388.
Rule, N. O., Ambady, N., Adams R. B. Jr., and Macrae, C. N. (2008). Accuracy and awareness in the perception and categorization of male sexual orientation. J. Pers. Soc. Psychol. 95, 1019-1028.

Russell, J. A., Weiss, A., and Mendelsohn, G.A. (1989). Affect grid: a single-item scale of pleasure and arousal. J. Pers. Soc. Psychol. 57, 493-502.

Shelp, S. G. (2002). Gaydar: visual detection of sexual orientation among gay and straight men. J. Homosex. 44, $1-14$.
Conflict of Interest Statement: The authors declare that the research was conducted in the absence of any commercial or financial relationships that could be construed as a potential conflict of interest.

Received: 22 February 2010; paperpending published:06 April 2010; accepted: 13 April 2010; published online: 07 May 2010.

Citation: Colzato LS, van Hooidonk L, van den Wildenberg WPM, Harinck F and Hommel B (2010) Sexual orientation biases attentional control: a possible gaydar mechanism. Front. Psychology 1:13. doi: 10.3389/fpsyg.2010.00013

This article was submitted to Frontiers in Cognition, a specialty of Frontiers in Psychology.

Copyright (c) 2010 Colzato, van Hooidonk, van den Wildenberg, Harinck and Hommel. This is an open-access article subject to an exclusive license agreement between the authors and the Frontiers Research Foundation, which permits unrestricted use, distribution, and reproduction in any medium, provided the original authors and source are credited. 